\title{
EL VAPOR EN EL LABORATORIO: UNA MEMORIA SOBRE LA EBULLICIÓN DEL ABATE NOLLET*
}

\author{
Manuel A. Sellés \\ Dpto. de Lógica. UNED
}

\section{RESUMEN}

En este artículo se presentan las investigaciones del abate Nollet sobre el agente responsable del fenómeno de la ebullición. Estas investigaciones constituyen un ejemplo destacado de las concepciones y métodos de la física experimental francesa a mediados del siglo XVIII, antes de su transformación en una física cuantitativa.

PALABRAS CLAVE: siglo XVIII, Francia, física experimental, Nollet, vapor.

\section{SUMMARY}

This paper presents the abbé Nollet'investigations about the agent causing the liquids boiling. This investigation are a case study about the conceptions and methods of the french experimental physics in the middle of the eighteenth century, before their transformation in quantitative physics.

KEY WORDS: $18^{\text {th }}$ century, France, experimental physics, Nollet, steam.

Uno de los aspectos más significativos del desarrollo de la ciencia en el siglo XVIII es la cuantificación que, a partir más o menos del tercer cuarto del siglo, comenzó a permear el estudio de fenómenos como los del calor, la electricidad o el magnetismo, que hasta ese momento habían sido objeto de un tratamiento cualitativo dentro de la física experimental. La etapa que precedió a estos cambios, salvo en el caso de la electricidad, no es bien conocida. Dentro de los estudios sobre el calor, por ejemplo, la historiografía tradicional arranca de las investigaciones de Joseph Black sobre los calores específicos y latentes, sin mencionarse apenas la etapa anterior. Por otra parte, esta historiografía, predominantemente anglosajona, ha prestado poca atención a los desarrollos de la física experimental ajenos a aquéllos que evolucionaron bajo el signo del newtonismo. Una figura particularmente desatendida es la del abate Nollet, pese a que lideró durante décadas la física francesa. Nollet tuvo la poca fortuna de sostener, frente al norteamericano Benjamin Franklin, una teoría de la

\footnotetext{
* Este trabajo está en deuda con el proyecto de investigación DGICYT PB 89-200.
} 
electricidad que fue superada por la de éste. ${ }^{1}$ Así, la física experimental francesa de la época ha sido más frecuentada desde el punto de vista de la vertiente espectacular y pública de la ciencia de la época que desde el de sus métodos y contenidos.

Otra razón para que esta etapa haya sido desatendida es la ausencia de logros: no hubo descubrimientos importantes. Y se entiende que no los hubo porque el camino emprendido era, por así decir, erróneo: la buena vía vendría por el desarrollo de conceptos que, como el de carga eléctrica o el de cantidad de calor, posibilitasen las mediciones al margen de los mecanismos concretos imaginados para explicar los fenómenos. Así, por ejemplo, T. S. Feldman, quien ha estudiado la historia de la meteorología — significativamente, sólo en la segunda mitad del siglo—, salda la época precedente con este comentario:

«La cuantificación de la física experimental constituyó una revolución en los métodos, estilos y actitudes. Los filósofos naturales de la primera paerte del siglo construyeron teorías cualitativas, en concreto, del vapor y de la evaportación, imágenes visualizables [pictorial images] tales como el lanzamiento de partículas de agua por partículas de fuego violentamente agitadas, o la expansión de partículas de agua en burbujas llenas de vapor por el calor. En los experimentos con el vapor procedieron de una manera asistemática, sin consideración a la precisión» ${ }^{2}$.

Estos calificativos son injustos. Como se verá en las páginas que siguen, Nollet estudió el vapor de agua de una manera bastante sistemática. Pero su objetivo no era el de realizar medidas o establecer relaciones cuantitativas, como sería el caso, poco después, de las determinaciones de los calores específicos y latentes. Ni tampoco los mecanismos visualizables imaginados para explicar los fenómenos dejaban de ser hipótesis a las que se concedía un mayor o menor grado de plausibilidad en función de su alcance explicativo ${ }^{3}$.

1 En este punto comparto la opinión de R. W. Home (1979), «Nollet and Boerhaave: A Note on Eighteenth-Century Ideas about Electricity and Fire», Annals of Science, 36, 171-76, esp. p. 171, y R. W. HOME (1985), «The Notion of Experimental Physics in Early Eighteenth-Century France», en PITT, J. C. (ed.), Change and Progress in Modern Science, Dordrecht, Reidel,107-131, esp. p. 108.

2 Feldman, T. S. (1983), The History of Meteorology, 1750-1800: A Study in the Quantification of Experimental Physics, Berkeley, Univ. of California, Ph. D., p. 6. (Tanto ésta como las restantes traducciones del artículo son mías).

3 Del mismo modo que hubo diversas variedades de newtonismo, las hubo también de cartesianismo. De hecho existió una tradición de física experimental cartesiana en la que, al compás de la interpretación del cartesianismo como un conjunto de hipótesis más o menos probables, desvirtuando la concepción inicial del maestro de un sistema fundamentado en ideas claras y distintas, los datos de la observación originaban hipótesis cuyas consecuencias se contrastaban posteriormente con la experiencia. Hipótesis cuyo grado de verosimilitud crecía con el número de hechos cuya explicación podían abarcar. Algo de esta evolución puede verse el estudio de P. MouY (1934), Le Développement de la Physique Cartésienne, 1644-1712, París, Vrin. Sobre el carácter probabilista del conocimiento de la naturaleza al otro lado del Canal puede verse B. J. SHAPIRO (1983), Probability and Certainty in Seventeenth-Century England, Princeton, N. J., Princeton Univ. Press. 
En las páginas siguientes estudio una memoria que el abate Nollet presentó a la Academia de Ciencias francesa en junio de 1748. La memoria es conocida por su explicación del fenómeno de ósmosis, al estudiar la difusión de los líquidos a través de una membrana animal ${ }^{4}$. Pero mi interés se centra en su investigación del fenómeno de ebullición; más concretamente, dicho en términos actuales, en su interpretación del cambio de estado que se produce durante la misma y en las características del vapor resultante. Naturalmente, Nollet no alcanza grandes conclusiones; pese a su aparente simplicidad, el estudio del vapor escondía importantes dificultades que sólo se irían superando en el siglo siguiente. Si bien es ejemplar por otros conceptos. En ella, Nollet describe paso a paso los experimentos y los razonamientos sobre sus resultados, dentro de una exposición ciertamente retórica, pero cuyo fin es la didáctica de los métodos de indagación del momento en física experimental. Y esto permite comprender el tipo de problemas al que se enfrentaba cualquier investigación en la disciplina. Pero antes de entrar en materia, haré algunos comentarios para enmarcar la cuestión, tanto desde el punto de vista metodológico como de lo que se pensaba en la época acerca del calor y de la evaporación.

\section{OBJETIVOS Y MÉTODO DE LA FÍSICA EXPERIMENTAL}

Tocaré sólo muy brevemente las materias mencionadas en el encabezado de este apartado, con el fin de enmarcar las experiencias de Nollet; un estudio llevado a cabo con alguna profundidad requeriría un trabajo específico que, por cuanto sé, todavía está por realizar ${ }^{5}$.

El programa de la física experimental consistía en inducir las leyes de la naturaleza a partir de los fenómenos, estudiados mediante la observación y el experimento. ${ }^{6}$ La denominación de «experimental» servía para distinguirla de la llamada «física sistemática», a la que se acusaba de intentar basarse en hipótesis indemostrables, sin que suministrase un verdadero conocimiento de las cosas, que para el físico experimental sólo podría adquirirse a través de los sentidos. En un principio, estos sistemas eran los de Aristóteles y Descartes, pero en el caso de Nollet se incluía también al de

4 TORLAIS, J. (1987), Un physicien au siècle des Lumières: l'Abbé Nollet, 1700-1770, Elbeuf-surAudelle, Jonas Editeur, (ed. orig. 1954), pp. 83-85.

5 El estudio clásico sobre estos temas es el de BRUNET, P. (1926), Les physiciens hollandais et la méthode expérimentale en France au XVIIIe siècle, París, Blanchard, que pese al tiempo transcurrido todavía puede leerse con algún provecho, si bien su opción de una influencia exclusiva de los físicos experimentales holandeses sobre la tradición francesa se ha puesto, con justicia, en entredicho.

6 No debe confundirse con lo que podríamos llamar el «programa docente» de la disciplina, en donde el protagonista era el experimento demostrativo, y en el que se buscaba exponer de una manera más o menos sistemática la filosofía natural de la época por medio de experimentos, recurriendo lo menos posible a las matemáticas, dado que por lo general el público no estaba familiarizado con ellas. 
Newton, que se había desarrollado hasta el punto de fundar la explicación de los fenómenos en atracciones y repulsiones microfísicas a distancia:

«No me presento aquí bajo los auspicios de ningún Filósofo: no son ni la Física de Descartes, ni la de Newton, ni la de Leibniz, las que me he prescrito seguir en particular: es, sin ninguna preferencia personal, y sin distinción de nombre, aquélla que me parece haber sido bien establecida por un acuerdo general y hechos bien constatados» ${ }^{7}$.

Dicho en términos generales, las otras dos escuelas de física experimental, la inglesa de Keill y Desaguliers y la holandesa de 'sGravesande y Musschenbroek, se declaraban abiertamente newtonianas (y se apoyaban en lo que manifestase Newton sobre la necesidad de fundarse en la inducción), haciendo intervenir en sus explicaciones una alternancia de atracciones y repulsiones entre las partículas de materia. La escuela francesa, sin embargo, tenía una tradición algo distinta que se manifiesta, con aún mayor claridad que en Nollet, en su predecesor Pierre Polinière quien, a la par que los físicos ingleses y holandeses, habría influido en Nollet. En general, dentro de la disciplina, al margen de la tendencia que se adoptase, el experimento se contemplaba como una verdadera herramienta de investigación capaz de permitir descubrir, por inducción o, como se decía entonces, por «analogía», las leyes de la naturaleza. Pero siempre se defendía la necesidad de las matemáticas, por más que éstas no apareciesen en los manuales de física experimental. Sin embargo, en la tradición francesa de Polinière y Nollet estas matemáticas no se consideraban una herramienta del todo apropiada, dado que se veía a la física como una disciplina esencialmente imprecisa $^{8}$. Lo que significa que, a diferencia de la posterior generación que configuraría una física experimental «exacta», cuanto menos en la tradición que representaba Nollet no se contemplaba la posibilidad de una manipulación matemática de relaciones funcionales establecidas entre magnitudes medibles.

Por otra parte, los físicos experimentales distinguían su disciplina de la historia natural por el hecho de que en ella no se aspiraba, simplemente, a una recopilación sistemática de experiencias, sino a la consecución de una estructura de principios, relacionados entre sí, y apoyados por los hechos. Dichos principios constituirían las causas de los fenómenos, y resultarían de la inducción. Y la indagación estaba sometida a ciertas reglas. Así, se insistía en la repetición de las experiencias, intentándolas de diversas maneras, para asegurarse del resultado. $\mathrm{Y}$ en tomar grandes precauciones antes de asegurar que algo era la causa de un fenómeno. Musschenbroek prescribía lo siguiente:

7 Nollet, J. A. (1754-65), Leçons de physique expérimentale, Amsterdam y Leipzig, vol. I, VXIII. En el vol. II, pp. 474, afirma que la atracción de Newton explica muy bien los fenómenos celestes, «Pero cuando se trata de fenómenos sublunares, de esos efectos que vemos desde más cerca, y cuyo examen nos resulta más fácil, la virtud atractiva es un Proteo que cambia a menudo de forma.»

8 HoME, R. W. (1985), pp. 124-25. 


\begin{abstract}
«No se deben pues admitir como causas más que aquéllas que indican manifiestamente los fenómenos de la Naturaleza. Estas [causas] serán verdaderas: $1^{\circ}$. si es constante que existen en la Naturaleza y si todos los fenómenos concurren en demostrar su existencia; $2^{\circ}$. si no solamente los fenómenos pueden ser deducidos [de ellas], sino además tienen una conexión necesaria con las causas; $3^{\circ}$. si los cuerpos sometidos a prueba y tratados de diferentes maneras nos indican constantemente las mismas causas de los mismos fenómenos; $4^{\circ}$. si no se pueden suprimir estas causas sin destruir los fenómenos mismos»9.
\end{abstract}

Otras dos importantes características de la física experimental consistían en su inmoderada fe tanto en los instrumentos empleados como en los hechos de la experiencia, y en su negativa a descarnar los fenómenos. Ambas están relacionadas, y se han interpretado a menudo diciendo que la física experimental no era predictiva, o bien que existía una conexión laxa entre las explicaciones y los fenómenos. La cosa es que para establecer un constructo matemático sobre un conjunto de fenómenos hay que reducir éstos a la geometría: el constructo es un modelo de la realidad, elaborado a base de eliminar de ésta los aspectos considerados más irrelevantes. Así, por ejemplo, un modelo de atmósfera la consideraría constituida por un elemento simple, el aire, y como tal homogéneo y formado por corpúsculos iguales. Para el físico experimental, sin embargo, el aire atmosférico era, en todo caso, una mezcla de dicha sustancia simple con, cuanto menos, polvo, vapores y exhalaciones, llegando a admitir incluso una diversidad de tamaños entre sus corpúsculos componentes (con lo que ya no sería tan simple). Y todo ello intervenía, o podía hacerlo, en los resultados del experimento, cuando éste se orientaba a buscar las causas de un fenómeno. El físico experimental abrazaba la realidad en una extensión en la que no podía hacerlo el matemático ${ }^{10}$. Por otra parte, el físico matemático podía predecir un efecto y someterlo a prueba diseñando un experimento orientado a mostrar inequívocamente la magnitud de tal efecto, desdeñando la contribución a dicha medida de otros secundarios que se verían como «causas de error» y se tratarían de reducir a límites aceptables; incluida en esto la teoría del instrumento de medida. Para el físico experimental, ajeno en general en una primera etapa a la búsqueda de relaciones matemáticas, no hay «causas de error»: todo forma

9 P. van Musschenbroek (1769), Cours de physique expérimentale et mathématique, trad. de Sigaud de la Fond, 3 vols., vol. 1, p. 15.

10 «Damos el nombre de Meteoros a todos los Cuerpos que están suspendidos en nuestra Atmósfera, que flotan, que son llevados y que se mueven. Comprendemos también bajo esta denominación todos los Cuerpos que se inflaman en la Atmósfera, todos los que se encuentran aislados, o que están mezclados con otros, los que se separan después de su unión, los que ascienden o los que descienden, en fin todos aquellos que producen algunos Fenómenos.» P. VAN MussChENBROEK (1739), Essai de Physique...avec une description de nouvelles sortes de machines pneumatiques et un recueil d'expériences, Leiden, 2 vols., vol. 2, p. 728. Algo más adelante manifiesta que todos estos «meteoros» se pueden mezclar de tan tan gran variedad de maneras para producir fenómenos diferentes, que «queda pues fuera de toda duda, que los meteoros deben producir un gran número de fenómenos, de los que nunca comprenderemos bien las causas, y sobre las cuales los filósofos no formularán nunca más que conjeturas». Ibid., p. 734. 
parte del fenómeno. Pero, análogamente al caso del físico matemático, cuyo experimento estará diseñado para mostrar o medir un efecto predicho por su modelo, el físico experimental interpretará el fenómeno que está ensayando a la luz de un marco teórico. Y en este sentido, aunque no haya dispuesto el experimento para mostrar un aspecto determinado en detrimento de todos los demás, verá por lo general lo que está preparado para ver.

\section{MATERIA, CALOR Y VAPOR}

Los físicos experimentales newtonianos, como Desaguliers y Musschenbroek, concebían la materia siguiendo las ideas que Newton expresó en su Óptica. Entendían que la materia constaba de átomos iguales y físicamente indivisibles, que por fuerzas de atracción se reunían en diverso número y configuración para formar las partículas de los cuerpos, si bien estas partículas de «primer orden» podían asimismo reunirse para formar otras de «segundo orden», y éstas a su vez otras de tercero, etc., dando lugar así a toda la diversidad que exhiben los cuerpos materiales, los cuales podían hallarse formados a la vez por partículas o corpúsculos de distinto orden. Aquí por corpúsculo debe entenderse la porción mínima de la materia de un cuerpo que conserva todas sus propiedades macroscópicas, de un tamaño tan pequeño como para resultar indiscernible. La forma de dichas configuraciones explicaba la diversa porosidad de la materia ${ }^{11}$.

Dentro de este esquema, la elasticidad de los fluidos se atribuía a fuerzas de repulsión. El modelo de aire que emplease Newton hipotéticamente en los Principia para deducir la ley de Boyle fue extendido por Desaguliers a los diversos fluidos en $1729^{12}$. Según éste, entre las partículas de materia existen fuerzas atractivas de muy corto alcance (las llama «atracción de cohesión» y sugiere que quizás varíen inversamente como la cuarta potencia de la distancia), pero en el caso de los fluidos elásticos actúan también fuerzas repulsivas de mayor alcance, que comienzan a operar y a predominar sobre las atractivas cuando, por el calor, la fermentación o cualquier otra causa, las partículas se ven separadas del contacto. De modo que los sólidos se pueden convertir en fluidos ${ }^{13}$. Éste será también el parecer de Musschenbroek ${ }^{14}$.

11 VAn MusschenBroek, P. (1739), vol. 1, pp. 41 ss.

12 Newton, I. (1987), Principios matemáticos de filosofía natural, Introd. y trad. de E. Rada, Madrid, Alianza, Libro II, Sec. 4, Prop. 23,pp. 494-97.

13 Desaguliers, J. T. (1729), «An Attempt to solve the Phaenomenon of the Rise of Vapours, Formation of Clouds and Descent of Rain», Philosophical Transactions of the Royal Society, $\mathrm{n}^{\circ}$ 407, 36, 6-22. Para un somero repaso de las ideas acerca del cambio de estado en Inglaterra, véase A. W. BADCOCK (1960), «Physics at the Royal Society, 1660-1800. I. Change of state», Annals of Science, 16, 95-115.

14 VAN MUSSCHENBROEK, P. (1739), vol. 1, p. 365. Frente a lo que afirman los cartesianos, que la liquidez se debe al movimiento de las partes y que, cesando éste, el fluido se convierte en sólido, argumenta que sus partes se encuentran en reposo. Si bien acepta que la experiencia muestra que diversos 
Nollet, que no es newtoniano, no se pronuncia sobre la divisibilidad indefinida de la materia, es decir, sobre la existencia de los átomos, pero naturalmente acepta la separación de la materia en partes muy pequeñas ${ }^{15}$. Conjetura que el estado natural de casi todos los cuerpos es la solidez, y que cuando son líquidos esto se debe a la penetración en ellos de una materia que, por su cantidad o por su acción, da a sus partes una mobilidad que rompe casi toda la adherencia entre ellas. Por ejemplo, el agua cesa de ser hielo en cuanto la materia del fuego lo penetra en cantidad suficiente. Respecto de las causas de la elasticidad de los sólidos, dice que no se conocen ${ }^{16}$.

En cuanto a la naturaleza del fuego, tanto Musschenbroek como Nollet siguen, en términos generales, la entonces influyente teoría de Boerhaave: el fuego constituye una materia muy sutil distribuida por todo el espacio, pero con un diverso grado de agitación que, al insinuarse dicha materia en los poros de los cuerpos, ejerce una acción disgregadora sobre la unión de las partículas de materia ordinaria, al comunicarles su movimiento ${ }^{17}$. En este sentido, Musschenbroek recoge dos mecanismos distintos mediante los cuales el fuego podría «evaporar» al agua. Por una parte, debido a su agitación, podría arrojar a las partes del agua fuera de su masa por las leyes de la percusión, si bien esto sólo lo menciona de pasada. Por otra, podría «rarificarlas» aumentando su volumen, tornándolas más ligeras específicamente que el aire, lo que explicaría su elevación en la atmósfera.

La evaporación a temperatura ambiente entraba dentro del campo de la meteorología, y ahí el interés se centró en el mecanismo por el cual el agua, con mayor peso específico que el aire, podía ascender en éste. Un mecanismo que obtuvo buena aceptación en la primera parte del siglo fue la llamada «teoría de las vesículas» propuesta por Edmond Halley en $1688^{18}$. Según esta idea, el calor expandía las partículas de agua en burbujas o vesículas llenas de alguna sustancia, un vapor o un aire o fluido rarificado, más ligero que el aire atmosférico. Otras teorías hicieron intervenir la adherencia mecánica —ésta fue la propuesta de Nieuwentyt—o, como recogiera Musschenbroek, la percusión producida por las partículas de fuego fuertemente agitadas.

Musschenbroek tiene sus dudas acerca de que una partícula de agua pueda dilatarse sin perder su integridad tanto como sería preciso para su ascenso, y parece inclinarse por la idea de que el fuego, al insinuarse en los poros de la partícula, siendo una sustancia muy elástica, y hallándose en mayor cantidad en ella que en el medio

\footnotetext{
menta que sus partes se encuentran en reposo. Si bien acepta que la experiencia muestra que diversos cuerpos no son fluidos más que cuando sus partes son separadas por la acción del fuego, y son puestas por él en movimiento, aunque piensa que este movimiento es accidental, pues no dejarían de constituir un fluido gracias sólo a la interposición de las partes del fuego. (pp. 369-71).

15 NOLLET, J. A. (1754-65), vol. 1, pp. 11-15.

16 Ibid., p. 125 y pp. 142-43.

17 VAN MUSSCHENBROEK, P. (1739), vol. 1, pp. 451 ss.

18 Y antes que él, en 1666, en Bolonia, por Urbano d'Aviso. Véase MidDlETON, W. E. K. (1966), A History of the Theories of Rain and Others Forms of Precipitation, Nueva York, Franklin Watts, p. 23.
} 
circundante, la eleva con él. Esto haría entrar en juego fuerzas de mayor alcance que darían cuenta de la elasticidad del vapor así formado. Dichas fuerzas repulsivas dependerían de la elasticidad de la sustancia del fuego, pues la elasticidad que muestra el vapor de agua cuando está encerrado es tanto mayor cuanto mayor es su temperatura. Dicha elasticidad se pierde al enfriarse (lo que explica, por ejemplo, que las nubes no se encuentren más calientes que el aire circundante), llegando el vapor a condensarse de nuevo en agua cuando pierde todo su fuego adicional, pues la misma elasticidad del fuego explicaría su tendencia a repartirse uniformemente por todas partes. Si bien considera que puede haber otras causas para el ascenso de los vapores, como la acción de los vientos o un mecanismo de disolución de las partículas de agua en el aire, producido por una cierta atracción entre el aire y el agua. Este mecanismo daría cuenta de la rápida dispersión de los vapores ${ }^{19}$.

Por su parte, Nollet no se desvía de la opinión común de que el calor es, cuanto menos, una causa importante de la evaporación. El calor, repartido por todo nuestro globo y su interior, mantiene en movimiento a las partes insensibles de todos los cuerpos. Este movimiento obligaría a las partes más sutiles de los cuerpos a abandonarlos. Aunque opina que el calor no debe ser la única causa de la evaporación, pues ésta todavía se produce, incluso en los inviernos fríos, en la nieve y en el hielo ${ }^{20}$. Además, en estos casos, e incluso a temperaturas más benignas, le parece que el calor no es suficiente, ni para inflar de modo conveniente las vesículas, ni para dilatar las partículas. Por ello cree que, una vez separadas de su masa por el calor, las partículas ascienden en el aire gracias a un mecanismo de disolución ${ }^{21}$.

Pero, en definitiva, ¿qué era, para estos físicos experimentales, un «vapor»? Según Musschenbroek, y con él todos los demás,

«Todo lo que se eleva en el aire desde la tierra es conocido bajo el nombre de Vapor o Exhalación. Los vapores están compuestos de partes acuosas y húmedas. Las exhalaciones están compuestas de las partes sutiles de todo tipo de cuerpos, tanto sólidos como fluidos, las cuales no son ni acuosas ni húmedas»22.

Caracterización que se puede remontar a Los meteorológicos de Aristóteles. Así pues, a mediados del siglo XVIII no se había llegado a elaborar todavía el concepto

19 VAN MusschenBROEK, P. (1739), vol. 1, pp. 430-31, y vol. 2, pp. 736 ss.

20 Nollet, J. A. (1754-65), vol. 2, pp. 110-11.

21 Ibid., vol. 2, pp.115-123. La teoría de la disolución, si bien propuesta anteriormente, cobraría una amplia aceptación gracias a la memoria de CHARLES LE ROY (1755), «Mémoire sur l'élévation et la suspension de l'eau dans l'air», Mémoires de l'Académie Royale des Sciences (París) (1751), 481-518, donde caracterizó el que denominó «grado de saturación del aire», que hoy llamamos «punto de rocío» (pues el «grado» de le Roy se refiere a la escala termométrica). Sobre la evolución de la teoría, véase W. E. K. MIDDLETON (1966), pp. 28-35. Es importante notar que, de acuerdo con esta teoría, el vapor disuelto en la atmósfera no ejercería ninguna presión en absoluto o, dicho en términos de la época, no tendría elasticidad.

22 VAN MUSSCHENBROEK, P. (1739), vol. 2, p. 734. 
de vapor, tal como hoy lo entendemos. Las partículas de agua que pasaban a la atmósfera seguían siendo, en el fondo, partículas de agua sin más, y por tanto específicamente más pesadas que el aire, por lo cual, como hemos visto, debía existir tanto un mecanismo responsable de su separación de la masa principal como de su elevación en el aire hasta una cierta altura (los cuales, se ha visto, no tenían por qué coincidir) ${ }^{23}$. Para lo primero había que vencer la cohesión entre las partes del agua, bien se debiese ésta al simple reposo relativo de las partes, o a la presencia de fuerzas atractivas.

Por otra parte, no había consciencia alguna de la perfecta transparencia del vapor. Las pequeñas gotitas de agua en suspensión que forman la niebla, así como las nubes o el «vapor» visible que se eleva de una olla en la cocción, se veían como un auténtico vapor. Al igual que ese otro vapor, invisible por su transparencia, del que estaba cargada la atmósfera, y que se ponía de manifiesto por la condensación cuando el aire entraba en contacto con una superficie fría ${ }^{24}$. Todo dependía de su grado de sutileza o, quizás, de su mezcla con otras sustancias.

\section{LA OBSERVACIÓN DEL AGUA HIRVIENDO}

El agua se evapora tanto a temperatura ambiente como cuando se pone a calentar en el fuego; en este último caso se sabía que, alcanzada la temperatura de $80^{\circ} \mathrm{R}$, ésta no se elevaba más. Un tercer caso era la ebullición a bajas presiones, dentro de la campana de una máquina neumática, que se efectuaba a temperaturas menores. El hecho de que en el primer caso la superficie libre del líquido permaneciese en calma y que en los otros se agitase a borbotones no establecía diferencia alguna respecto al mecanismo de la evaporación, que como se vio se adjudicaba fundamentalmente a la acción del fuego sobre las partes del agua, atribuyéndose la agitación de su superficie bajo la campana neumática al desprendimiento del aire disuelto, y en el caso de hallarse en un recipiente al fuego al aire libre, a éste y a la posterior acción del fuego.

Nollet presenta tres argumentos para refutar la creencia de que la agitación de la superficie de los líquidos al hervir se debe exclusivamente al aire disuelto en ellos. El primero, que las experiencias de Guillaume Amontons, de Stephen Hales y de él mismo muestran que la cantidad de aire disuelto en el agua y su dilatación a la temperatura de ebullición no bastan para explicar el fenómeno. El segundo, que el agua purgada de aire, así como otros líquidos con diversa capacidad para disolverlo, borbotean al hervir con la misma intensidad hasta que se evaporan por completo. El

23 Lo cual era ciertamente muy incómodo a la hora de explicar las variaciones del barómetro con el estado del tiempo, pues, contrariamente a lo que se observaba, en el mal tiempo el peso de los vapores debía sumarse al del aire, haciendo subir la columna barométrica.

24 Por ejemplo, Musschenbroek habla, en un momento dado, de la elevación de vapores en el aire a consecuencia de la fina división de las partículas en la base de una cascada alta. Ibid., vol. 2, p. 740. 
tercero, finalmente, que lo que sale de una eolipila en la que se ha introducido agua, cuando se calienta, no es un chorro de aire (lo que, según dice, presentaban como prueba decisiva los partidarios de la explicación que está refutando), sino de «vapor dilatado del agua», como se comprueba al no verse salir burbujas cuando se sumerge su extremo en agua fría (Ilustr. 1, fig. 2).

De hecho, se sabía que no era exclusivamente aire. Por ejemplo J. T. Desaguliers había dejado bien sentado que lo que sale de la eolipila es vapor de agua, como se puede comprobar, decía, poniendo la mano cerca de su boca, y viendo cómo el agua se condensa en ella ${ }^{25}$. Por otra parte, sin embargo, afirmaba que la ebullición que se producía en el agua caliente introducida en la campana de la bomba de vacío cuando se extraía el aire se debía al aire disuelto ${ }^{26}$. Nieuwentyt, hacia la misma época, creía que la ebullición producida bajo una campana neumática era producida por el fuego abandonando el agua. ${ }^{27}$ Musschenbroek, aun afirmando que la temperatura de ebullición del agua depende de la presión, será de la misma opinión, afirmando que en la campana neumática el agua se descarga tanto más del aire disuelto cuanto más caliente está. En otra ocasión el mismo Desaguliers también afirmará que el vapor generado en la máquina de Newcomen no tenía nada que ver con aire disuelto en el agua, puesto que dicho vapor se podía condensar de nuevo en agua ${ }^{28}$. Parece, pues, que sobre estos extremos existía cierta confusión, aunque quizás no tanta como Nollet querría hacer creer $^{29}$.

En fin, el primer experimento que presenta Nollet consiste en la simple observación de la ebullición del agua en un recipiente transparente, por el calor de carbones bien encendidos (Ilustr. 1, fig. 3) 3 $^{30}$. Nota que, en una primera etapa, a los $30^{\circ} \mathrm{R}$, aparecen burbujas en el fondo del recipiente, que engrosan a $\operatorname{los} 40^{\circ} \mathrm{R}$ formándose también en las paredes, y desaparecen a los $70^{\circ} \mathrm{R}$. Se trata, afirma, de burbujas formadas

25 Desaguliers, J. T. (1719), A System of Experimental Philosophy, Londres, p. 11.

26 Ibid., pág. 132. Añade que mediante este procedimiento se puede extraer casi todo el aire disuelto en el agua.

27 DYMENT, S. A. (1937), «Some eighteenth century ideas concerning aqueous vapour and evaporation», Annals of Science, 2, 465-473; pp. 466-67.

28 Desaguliers, J. T. (1729), p. 17

29 En este contexto parece haberse olvidado una antigua experiencia de ebullición en el vacío que realizó Robert Boyle. La recogió en BOYLE, R. (1660), New Experiments Physico-mechanicall, Touching the Spring of the Air, and Its Effects (...), Oxford, en donde ya atribuyó el efecto a los vapores excitados por el calor: «Que al retirarse la presión del Aire que la ocupa, bien a los Corpúsculos Igneos, o más bien a los Vapores agitados por el calor en el Agua (los cuales, como hemos notado anteriormente tocando el Agua rarificada de una Eolipila, se manifiestan capaces de un Poder Elástico) se les permite expandirse poderosamente en el Recipiente evacuado; y, en su tumultuosa Dilatación elevan (como el Aire acostumbra a hacer) la parte superior del Agua, y tornándola en Burbujas, hacen que el Agua hierva.» (pp. 389-90).

30 De hecho, la memoria de Nollet contiene más experiencias de las que aquí se comentan, que son las que parecen más significativas Por esta razón algunas de las figuras de las láminas no encuentran correlato en el texto. 


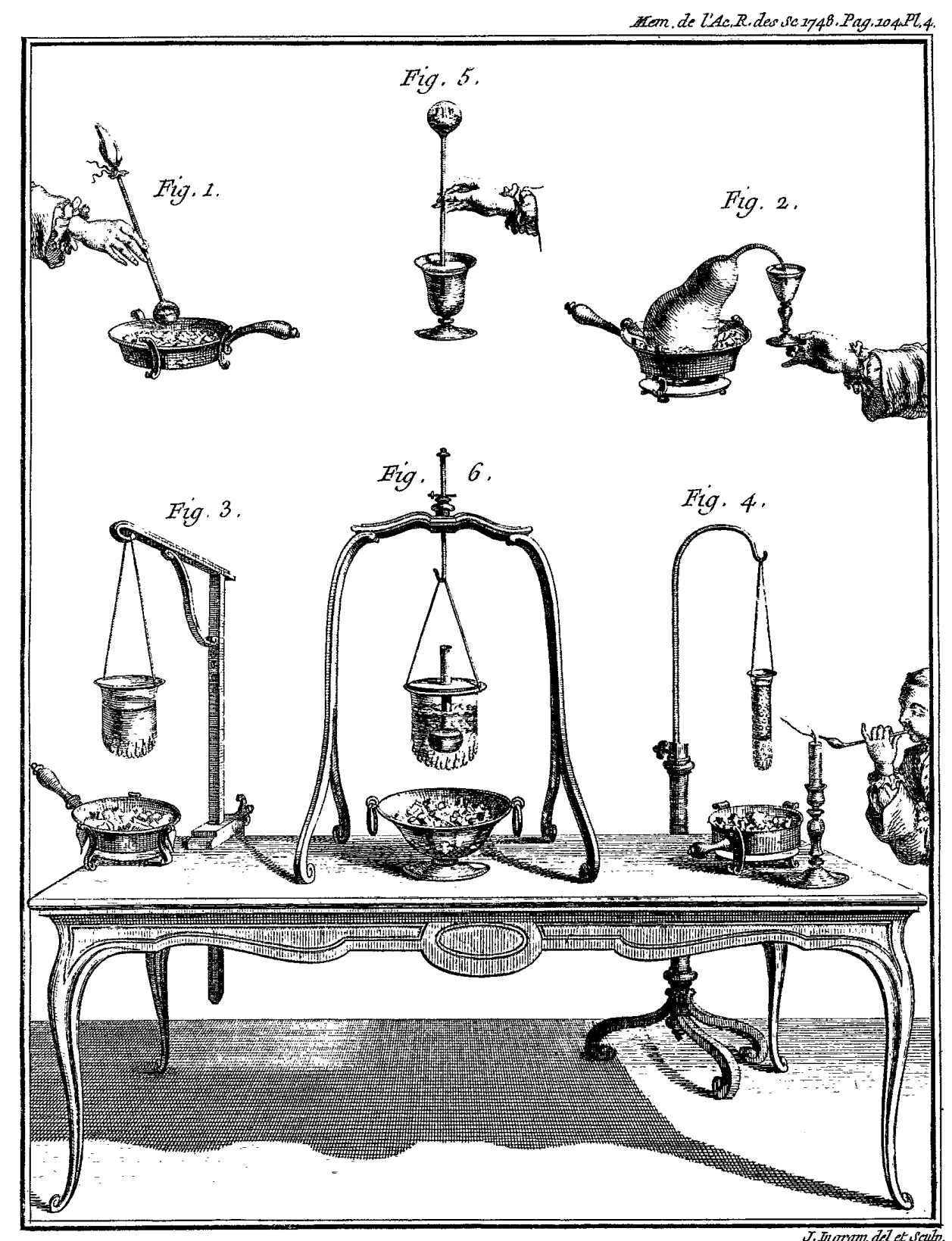

Ilustración 1 
por el aire disuelto. En ese momento, cuando estas burbujas desaparecen, ve elevarse del fondo «un vapor muy sutil» cuyos «filetes» o «rayos» se tornan más perceptibles con el aumento de la temperatura, hasta que acaban por invadir toda la masa del agua. Sus partes le parecen a primera vista como granos o burbujas imperceptibles. Despojada el agua del aire que contenía, estos filamentos de «vapor sutil», que surgen de la parte del recipiente más expuesta al calor, que turban la limpidez del agua y se mezclan con ella, le parecen consistir de «la materia misma del fuego, si no del fuego puro y desprendido de toda otra sustancia, cuanto menos de fuego unido a las partes más sutiles de su alimento» ${ }^{31}$. Aunque presenta esto como una conjetura, aquí Nollet, quien como Musschenbroek y tantos otros aceptaba la teoría de Boerhaave, está viendo lo que esperaba ver, a la materia del fuego abriéndose paso a través del recipiente y del agua, para terminar escapando por su superficie:

«imagino que estos rayos de materia ígnea, saliendo de los carbones ardientes como de su fuente, dilatan los poros del vidrio, atravesando su espesor, y se esparcen en el agua como un vapor, que se deja ver, quizás menos por su sombra que por las diferentes refracciones que hace sufrir a la luz en un medio transparente del que altera la homogeneidad (...).»32

Esta materia del fuego, que surge con mayor abundancia y velocidad con el aumento del calor que ella misma produce, escapa tanto por las paredes del vidrio como por la superficie libre del agua. Aún arrastradas por el fuego, las partículas de agua no pueden atravesar los poros, demasiado estrechos, de las paredes del recipiente, pero no encuentran este obstáculo en la superficie del líquido, por encima de la cual forman un vapor visible, más o menos espeso.

En este punto, Nollet imagina que la ebullición no dependería más que de que los rayos del fuego que penetran en el recipiente adquieran una cierta densidad, o quizás una cierta velocidad. Ésta es, más o menos, la idea de Musschenbroek, quien afirmaba que el agua no podía contener en sí más que una determinada cantidad de fuego, de modo que el exceso levanta el agua, produciendo la ebullición ${ }^{33}$.

La cautela con la que expone la hipótesis anterior sobre la causa de la ebullición tiene algo de retórico, pues a continuación él mismo presenta un hecho que la refuta:

31 Nollet, J. A. (1752), «Recherches Sur les Causes du Buillonnement des Liquides», Mémoires de l'Académie Royale des Sciences (París), (1748), 57-104; p. 66.

32 Ibid., p. 66-67.

33 VAN MussChENBROEK, P. (1739), vol. 1, p. 426. Años más tarde, Joseph Black manifestará que ésta era una opinión general. Y objetará que, si cuando el agua llega a la ebullición está saturada de fuego y basta una pequeña cantidad para evaporarla, ¿por qué no se evapora toda de golpe? MCKIE, D. y DE V. Heathcote, N. H. (1975), The Discovery of Specific and Latent Heats, Nueva York, Arno Press (ed. orig. 1935), p. 20. Los experimentos sobre este extremo le llevarán al descubrimiento del calor latente de vaporización. Antes que Black, J. Rowning habría opuesto un argumento similar a la teoría de Desaguliers. DYMENT, S. A. (1937), p. 468. 
si la idea va bien para los líquidos, no sucede lo mismo con algunas materias licuefactadas, como el estaño y el plomo, que no muestran el fenómeno de ebullición por más que se las caliente: simplemente se «queman». Y acto seguido relata que, en un primer momento, atribuyó esta diferencia de comportamiento al peso de las partículas; siendo las de los metales más pesadas, no pueden ser elevadas por el fuego. Él mismo, a continuación, refuta esta idea: el mercurio, que después del oro pesa más que cualquier otro metal, sí que hierve ${ }^{34}$. He aquí toda una lección sobre el método de la física experimental: la observación, guiada por el recto razonamiento, conduce a la formulación de hipótesis plausibles. Pero allí donde muchas observaciones confirmatorias sólo aumentan el grado de certidumbre de una hipótesis, un solo hecho en contra las derriba. La solución es, naturalmente, realizar nuevos experimentos para examinar más a fondo la cuestión ${ }^{35}$.

\section{LO QUE SUCEDE EN EL FONDO DEL RECIPIENTE}

Dado que el fenómeno parece originarse en el fondo del recipiente, donde el grado de calor es mayor, Nollet reproduce la situación calentando una placa de hierro y echando sobre ella gotas de agua. Éstas hierven y se disipan. Ahora mezcla el agua con una sustancia más densa, goma arábiga. Entonces ve formarse ampollas, que se hinchan y revientan exhalando una bocanada de vapor. Con la goma arábiga ha puesto de manifiesto el proceso que se producía en las gotas de agua: las ampollas estarían formadas por partículas de agua elevadas y dilatadas desde la superficie del hierro por el calor, y momentáneamente retenidas por una película de la misma sustancia que, ahora más viscosa con la adición de la goma arábiga, se rompía con más dificultad.

En este punto Nollet expone una teoría de la ebullición — de la ebullición, no del cambio de estado - que hoy consideraríamos, salvando las distancias, bastante aceptable. El calor recibido por la parte del recipiente más expuesta al fuego es más del que puede admitir, sin evaporarse, el líquido que está en contacto con ella. Así, se convierte en una burbuja de vapor dilatada, cuyo volumen aumenta hasta que se desprende, reteniendo desde ese momento su grado de calor y haciendo borbotear al

34 Un resultado similar había sido hallado poco antes al otro lado del Canal por George Martine, y publicado en sus Essays Medical and Philosophical (Londres, 1740). Halló que, entre los materiales ensayados, y después del aire, el mercurio era el que más rápidamente se calentaba y se enfriaba. McKIE, D. y DE V. HeATHCOTE, N. H. (1975), pp. 14-15. Más tarde Black lo explicaría en términos de las diferentes capacidades para el calor.

35 Tal como lo expresaría Desaguliers, refiriéndose a la necesidad de examinar las ideas recibidas de otros: «This Examen is to be made by Suppositions, which we may rely upon, when they agree with Experiments; but if only one Experiment is contrary to any Supposition, that Supposition must be rejected, and a new one made, till we find that it agree with all other Experiments.» DESAGULIERS, J. T. (1719), p. 2. 
líquido al romperse en su superficie. El lugar que deja libre la burbuja al desprenderse de la superficie del recipiente lo ocupa nuevo líquido, que se evapora a su vez. De este modo, el «vapor sutil» que vio anteriormente antes de iniciarse la ebullición consta de pequeñas burbujas que, en un primer momento, se condensan al internarse en una masa de fluido todavía demasiado fría. La transparencia de estas burbujas se explica por la sutileza y homogeneidad del vapor de agua que las compone. En el caso de los vapores acuosos en la atmósfera, que no se perciben con tanta limpidez, ello se debe a su mezcla heterogénea con el aire.

Así pues, tal como se presenta en los pasos seguidos por Nollet, el método de indagación consistiría en:

a) Obtención de un hecho «crudo» a través de la observación.

b) Formulación de una explicación «plausible» dentro del marco de las concepciones aceptadas.

c) Extensión de dicha explicación a todos los fenómenos de la misma índole.

Si no aparecen excepciones, el hecho o fenómeno quedaría explicado. Si no es así,

d) Realización de nuevas observaciones orientadas a iluminar los puntos problemáticos: nueva recopilación de hechos.

e) Modificación de la explicación inicial, efectuando el mínimo de conjeturas adicionales posible.

f) Extensión de dicha explicación al mayor número posible de fenómenos, justificándose con ello las conjeturas adicionales.

A estas alturas de la exposición que hace Nollet en su memoria, nos hallamos en este último apartado (f). Comprueba que, cuanta mayor disposición tiene una sustancia a evaporarse, menos calor se necesita para hacerla hervir. Lo que en cierto modo explica el comportamiento anómalo del mercurio respecto de otros metales, pues se evapora con facilidad. La presencia, o ausencia, de ebullición se explica en términos de la resistencia a la formación de vapores expansibles. Esta resistencia proviene del peso del líquido - la presión - en el lugar donde comienza la ebullición, y de la adherencia de las partes de la sustancia, que el fuego tiende a vencer. Esto explicaría, además, por qué una sustancia no aumenta su temperatura cuando comienza a hervir, pues entonces, «quedando victoriosa la acción del fuego, ya no es susceptible de acrecentamiento» ${ }^{36}$. Pues la acción del fuego se realiza a proporción de la resistencia que encuentra en las diversas sustancias.

Si el peso del líquido en el lugar donde se inicia la ebullición es determinante del comienzo de ésta, también lo será el peso de la atmósfera sobre la superficie del líqui-

36 NOLLET (1752), p. 80. 
do, y esta consideración, manifiesta Nollet, hubiese llevado a predecir un hecho que Fahrenheit encontró por azar: que a mayor presión atmosférica, mayor calor necesita una sustancia para llegar a la ebullición. Y la inversa: que en el vacío necesita para hervir un calor menor. Lo que le lleva a experimentar con la máquina neumática.

\title{
EXPERIMENTOS EN EL VACÍO.
}

El montaje experimental de Nollet consiste en un recipiente con agua puesto al baño maría, cuya boca está unida por un tubo sellado con la campana de una máquina neumática, en cuyo interior ha situado un barómetro (Ilustr.2, fig. 7). Hace el vacío en la campana — llega hasta 3,5 pulg. de $\mathrm{Hg}$ - y calienta el agua. Todo sucede como antes: primero se liberan burbujas de aire, que apenas repercuten en el nivel del barómetro, y luego el agua hierve durante un rato. El agua está a $25^{\circ} \mathrm{R}$, el barómetro sube 2 líneas y en la campana de la máquina se extiende un «vapor cálido». Al bombear para sacar el aire desprendido en la ebullición, el mercurio, que está a 5,5 líneas del nivel inicial, no baja por más que bombea. La conclusión que extrae es sorprendente: el resorte del vapor, a diferencia del caso del aire, no se puede debilitar por el juego de la máquina neumática:

\begin{abstract}
«un calor bastante mediocre convierte en vapores la humedad que se encuentra encerrada en el recipiente, y desde ese momento el vacío ya no se hace tan perfectamente, sea porque la materia del fuego unida a partículas de agua, o de alguna otra sustancia muy paerecida, forma un fluido cuyo resorte no puede ser debilitado, como lo es el del aire común, por el juego de la bomba, sea porque el mismo aire cargado de vapor, aunque adquiera algún nuevo grado de resorte, se torna menos apropiado para extenderse por el tubo estrecho de la llave, donde reinan siempre otros vapores grasos y más groseros» ${ }^{37}$.
\end{abstract}

Lo que explica los distintos grados de vacío que se pueden obtener en la máquina neumática en distintas épocas del año, algo que él mismo había estudiado en una memoria presentada a la Academia algunos años antes ${ }^{38}$.

37 NOLLET (1752), p. 85. Lo que parece verosímil es que la presión en el interior de la campana no baje por el continuo aporte de vapor del agua hirviendo.

38 NolLET, J. A. (1742), «Sur la Vapeur qu'on apperçoit dans le Récipient d'une Machine Pneumatique, lorsqu'on commence à raréfier l'Air qu'il contient», Mémoires de l'Académie Royale des Sciences (París) (1740), pp. 243-253. Constató allí que, a las dos o tres primeras emboladas, cuando el aire comienza a rarificarse, aparecía en el recipiente un vapor más o menos espeso que oscurecía su interior y que, tras algunos pequeños movimientos en forma de circunvoluciones, se precipitaba en la parte inferior del recipiente. Lo atribuye a la condensación de los vapores de que está cargado el aire, producida por una disminución del resorte de éste. En sus experiencias hubiera podido constatar la necesidad de núcleos de condensación, algo que sólo se pondría de manifiesto en 1875. Nollet encontró que se producían menos vapores cuando el experimento se realizaba en una habitación limpia que cuando se efectuaba en su laboratorio. Véase MidDLETON, W. E. K. (1966), pp. 172-173. 
MANUEL A. SELLÉS

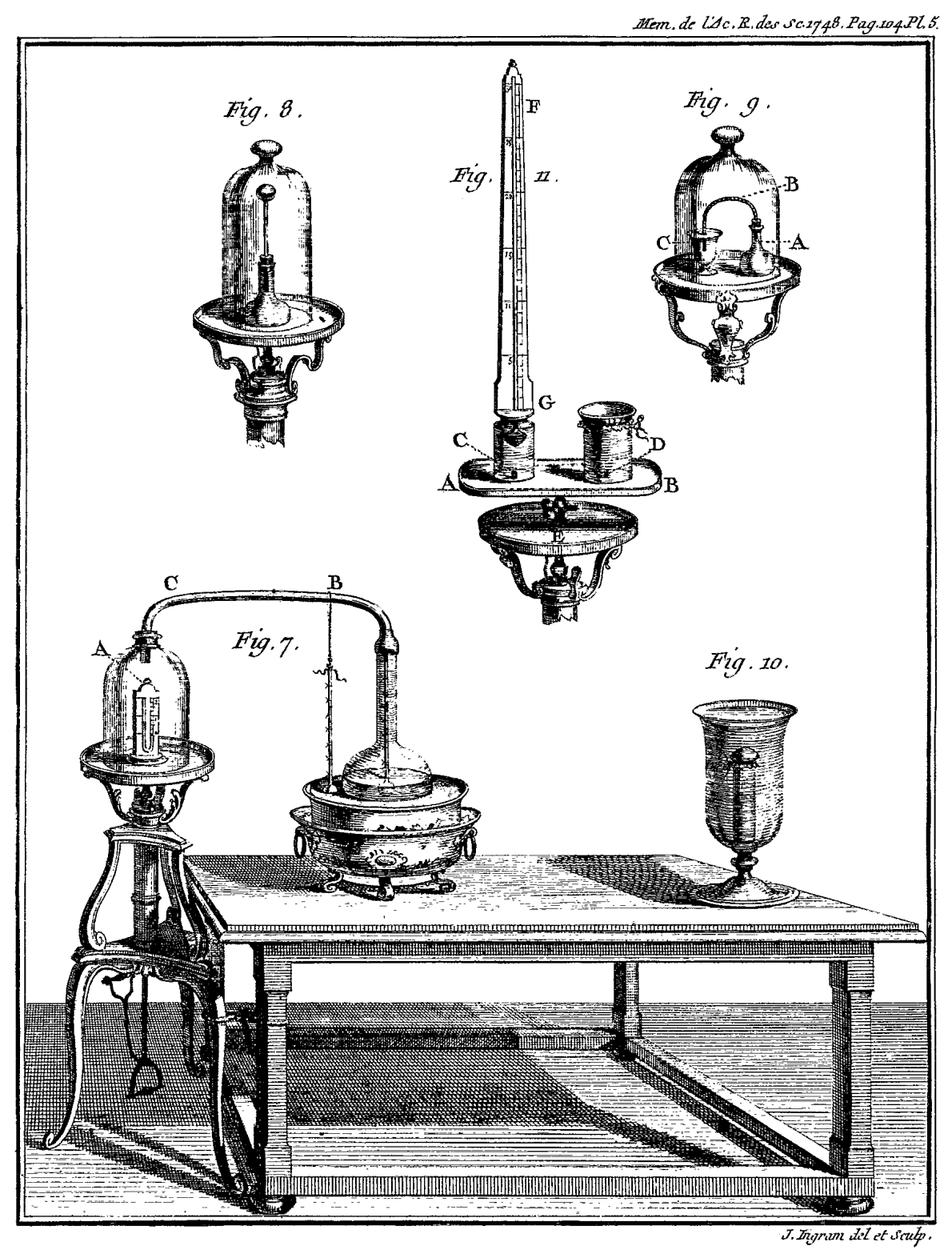

Ilustración 2 
Creyendo constatar un hecho, Nollet introduce aquí una hipótesis nueva, según la cual podrían existir dos tipos de «resorte», uno propio del aire y otro del vapor. Pero se corrige inmediatamente pues, de hecho, logrará hacer bajar el barómetro 4 líneas enfriando la campana. Lo que le indica que el aumento de presión estaba en realidad causado por:

«un vapor dilatado que suple en parte el resorde del aire debilitado por la rarefacción» ${ }^{39}$.

Con esta conclusión, parecería que Nollet podría haber anticipado en cierta manera - salvando, naturalmente, las distancias - un descubrimiento importante, la ley de las presiones parciales que enunciará Dalton medio siglo después. Pues parece asumir que los resortes del aire y del vapor actuarían independientemente, sumándose (cuanto menos en el interior de la campana de la máquina neumática). Pero un nuevo y extraño fenómeno interfiere, y no realiza posteriores indagaciones. Cuando enfría todos los recipientes con esponjas mojadas, encuentra al mercurio del barómetro 3 líneas por debajo de su nivel (el que tendría si la presión atmosférica fuese justamente nula), efecto que perdura durante alrededor de un minuto, tras el cual el mercurio sube lentamente a 3 líneas por encima, quedando así un poco más bajo que cuando hizo el vacío para iniciar la experiencia. De modo que los que atraen la atención de Nollet son dos sucesos inesperados:

1) El estacionamiento del mercurio por debajo de su nivel en el vacío, y

2) que el aire se encuentre más rarificado después de haberse condensado el vapor de lo que había podido conseguirse antes de que este vapor viniese a unírsele.

El segundo hecho se puede explicar diciendo que el vapor, al condensarse,

«se ha apropiado y ha como fijado una parte del aire con el cual se había mezclado, lo que habría disminuido en otro tanto la cantidad de aire elástico que había quedado en el recipiente $(\ldots) . » 40$

Pero el otro efecto es más difícil de explicar porque, como hace notar Nollet, se precisa de una «fuerza positiva» para hacer bajar el mercurio en una de las dos ramas, cuando, antes de descender, el mercurio la llenaba totalmente y no parecía haber allí otra cosa.

En realidad, el fenómeno no le resultaba nuevo, pues dice haberlo observado por primera vez cinco años atrás. El efecto es siempre patente en dos de sus barómetros de prueba (sifones invertidos, una de cuyas ramas está cerrada y llena de mercurio).

\footnotetext{
39 Nollet, J. A. (1752), p. 87.

40 Ibid., p. 87.
} 
En uno de ellos el tubo de vidrio es más grueso y el canal más estrecho que en el otro, siendo siempre el descenso del mercurio mayor en este último. Ha realizado experiencias, comprobando que estos instrumentos, cuando se sitúan en el interior de un recipiente que no contiene nada de «vapor cálido», se comportan igual que los demás. El efecto, evidentemente, proviene de la presión ejercida por algún fluido en la rama del barómetro que se considera vacía.

Antes de profundizar más en la cuestión, termina la experiencia, comprobando que la temperatura a la que se produce la ebullición depende de la presión en el interior de la campana. Deduce:

«que los licores hierven en el vacío por la misma causa que les hace hervir bajo el peso de la atmósfera; es siempre, por lo que creo, una pequeña porción de la masa que se convierte súbitamente en vapor, y que levanta al resto (...).»41

Sucede que, al disminuir la presión, el grado de fuego, que antes no tendría fuerza para vencer el peso de la masa de agua o la adherencia de sus partes, resulta suficiente para excitar las bocanadas de vapor que producen la ebullición. Y éstas,

«o son algo distinto del aire, o son otro aire distinto al que se extrae del recipiente por medio de la bomba.» 42

\section{EL ESTUDIO DEL VAPOR.}

El siguiente paso es recoger este fluido para tratar de analizarlo. Toma un pequeño matraz, lo llena por completo de agua tibia, lo invierte y sumerge su cuello en otro recipiente también lleno de agua, de tal modo que el conjunto, después de la inversión, queda perfectamente lleno. Lo coloca bajo la campana de la máquina y hace un vacío moderado, el suficiente para iniciar la ebullición (Ilustr. 2, fig. 8). Al cabo de un tiempo, el agua del matraz ha descendido notablemente. Entonces deja entrar aire en la campana, y cuando el agua se enfría a la temperatura ambiente encuentra un pequeño segmento de la esfera del matraz ocupado por aire. Le parece que esta porción responde bien al aire que podría haberse hallado disuelto en el agua, pero totalmente insuficiente para dar cuenta del volumen de las burbujas que ha observado a lo largo de toda la ebullición.

El siguiente paso es tratar de hacer visible al vapor haciéndolo pasar a través de agua. Toma un recipiente con agua tibia que ya había hervido, y lo comunica mediante un sifón con otro recipiente que contiene agua más fría en las mismas condiciones

\footnotetext{
41 Ibid., p. 90
}

42 Ibid., p. 90 
(Ilustr. 2, fig. 9). Introducidos en la campana, cuando comienza la ebullición ve llenarse al sifón de «vesículas transparentes» que pasan continuamente al otro recipiente mientras dura la ebullición. Aunque aparentemente parezcan compuestas de aire con una envoltura de agua, por lo visto anteriormente piensa:

«que, en el fondo, esta especie de soplo bien podía no ser más que un vapor flatulento, es decir, una pequeña porción del licor rarificada, y llevada por un fluido más sutil que el aire común, siempre presto a lanzarse en el recipiente desde el momento en que hay un vacío bastante perfecto, o que la masa fluida que lo encierra no le opone una coherencia de partes demasiado difícil de vencer» ${ }^{43}$.

Para examinar más atentamente las burbujas, pone agua tibia en un recipiente, y la cubre con unos tres dedos de «espíritu de trementina». Cuando comienza la ebullición y las burbujas pasan a través del mismo, percibe que al romper en su superficie se produce una pequeña lluvia muy fina que vuelve a caer, y que interpreta como la película acuosa que había servido de envoltura a dichas burbujas. Otras burbujas, que no llegan a alcanzar la superficie, vuelven a caer hasta la superficie del agua, donde disminuyen poco a poco de volumen hasta desaparecer totalmente. Entre tanto, la campana de la máquina se llena siempre de un vapor abundante. Lo que le lleva a creer:

«que la ebullición de los licores en el vacío, está causada por bocanadas de vapores, que un fluido más sutil que el aire común eleva y dilata bruscamente» ${ }^{44}$.

He aquí, pues, que separa al «vapor» del fluido que lo dilata, sobre cuya naturaleza afirma prudentemente no poder hacer más que conjeturas:

«podría tratarse de la materia del fuego que penetra, como se sabe, a los cuerpos más duros, así como a los más fluidos, y que se detiene sin embargo, en los unos y en los otros, hasta que una causa la determina a pasar de dentro a fuera: esta causa, en el caso del vacío, sería el mismo vacío, por el cual el licor, desprendido de toda presión exterior, se torna libre de obedecer a la fuerza expansiva del fuego que encierra» ${ }^{45}$.

Sin embargo, esta materia también podría ser la parte más sutil de la atmósfera, una parte tal que los cuerpos más compactos nunca fuesen totalmente impenetrables para ella. Y ésta es, curiosamente, la explicación por la que se inclina.

43 Ibid., p. 94

44 Ibid., pp. 94-5.

45 Ibid., p. 95. 


\section{EL COMPONENTE SUTIL DEL AIRE}

En la primera de sus dos conjeturas sobre la naturaleza del vapor, Nollet anticipa de alguna manera la teoría calórica de los gases que años más tarde defendería su compatriota Lavoisier. Siendo la base el agua, la elasticidad vendría suministrada por la cantidad de materia del fuego - Lavoisier lo denominaría calórico- en combinación con ella. Pero, sin embargo, prefiere recurrir a un hipotético componente del aire.

Pero Nollet no se lo saca de la manga; en realidad, tal componente sutil del aire ya había sido propuesto en relación con otros fenómenos. En este sentido, cita una serie de experiencias con barómetros realizadas en la Academia de Ciencias de París en los primeros años del siglo, la investigación de Huygens sobre la suspensión anómala, y las ideas de Dortous de Mairan sobre la aurora boreal.

En las experiencias realizadas en la Academia de Ciencias de París se estudió el hecho de que en distintos barómetros el mercurio ascendiese a alturas distintas. Se barajaron diversas hipótesis, entre ellas la supuesta existencia de una materia intermedia entre el éter y el aire que podría penetrar los poros del vidrio, ejerciendo presión en la rama del barómetro aparentemente vacía. También se barajó la idea de una dilatación de los restos de espíritu de vino que, pese a todas las precauciones, hubiesen podido quedar en el tubo; o del efecto del aire disuelto en el mercurio. En cualquier caso, el problema no se pudo acabar de resolver ${ }^{46}$. Los barómetros comenzarían a funcionar bien cuando, a partir del primer cuarto de siglo, se generalizase la práctica de hervir el mercurio en el interior del tubo, lo que además de producir su secado desprendía el aire contenido en el mercurio ${ }^{47}$. Nollet conocía bien estas precauciones, y manifiesta haberlas aplicado. De modo que no parece posible discernir la causa del fenómeno. Aunque, por otra parte, es muy curioso que el efecto se manifieste predominantemente en dos de ellos. Quizás pudiese deberse a alguna peculiaridad debida a su fabricación, que tampoco es fácil de imaginar.

El estacionamiento anómalo era una experiencia ya antigua y bien conocida, aunque no bien explicada. Fue investigada por Huygens en 1661, y fue ampliamente difundida tras su publicación en 1672. Fue repetida por Boyle, Hooke y Amontons. La experiencia era simple: si se llena un tubo con agua y se invierte en el interior de una campana neumática, éste se vaciará, al no haber presión que lo sostenga; pero si

46 Véase SELlÉs, M. A. (1995), «La ley de Amontons y las indagaciones sobre el aire en la Academia de Ciencias de París (1699-1710)», Asclepio, XLVII (1), 53-80.

47 Un cuarto de siglo después, J. A. De Luc manifestará que, para entonces, aún barómetros de muy distinta fabricación no se desvían ni en una décima de línea. No obstante, suscribe la idea de la permeabilidad del vidrio a un fluido que por su peso, o por su elasticidad, es responsable de fenómenos como la adherencia -muy superior a la que explicaría la presión señalada por el barómetro- de cuerpos pulidos, y que constituiría una parte esencial de la atmósfera. DE LUC, J. A. (1772), Recherches sur les modifications de l'atmosphère, 2 vols., Ginebra, vol. 1, pp. 36-38. 
se purga el agua del aire disuelto en ella, el agua se sostendrá hasta que se introduzca en el tubo la más mínima burbuja de aire. La explicación de Huygens, que no dejó de ser debatida, era que existía una sustancia, más sutil que el aire ordinario, que permea a los cuerpos, y particularmente al vidrio, y que ejercía una presión aun en el interior de la campana. Si bien el mismo Huygens era consciente de las dificultades de esta explicación, pues para que funcionase el fluido no tendría que permear al agua, ni al vidrio del extremo superior del tubo ${ }^{48}$.

Por su parte, en su teoría de la aurora boreal Mairan, convencido de que el fenómeno era atmosférico, tuvo que extender la atmósfera de la Tierra bastante más lejos de lo que se pensaba, lo que argumentó suponiendo que el barómetro sólo reflejaba una parte del peso de la atmósfera, aquél debido a un aire más grosero, incapaz de penetrar por los poros del vidrio ${ }^{49}$

A estos argumentos, Nollet añade dos observaciones. La primera es que, cada vez que ha hecho el vacío en una campana neumática cuyo interior estaba empañado por algún vapor, a medida que actuaba la bomba éste se disipaba. Si la campana es permeable a un aire sutil, al extraer el aire más grosero esta materia sutil penetra por los poros del vidrio, arrastrando a las partículas de agua que se han fijado allí50. Pero, como él mismo se objeta, si este aire sutil puede pasar a través del vidrio, ¿por qué no lo hace igualmente a través del agua que lo recubre? La solución es recurrir a un diverso tamaño de las partes del aire sutil y a carencias de alineación de los poros del agua. Lo cual se ve apoyado por la segunda observación ${ }^{51}$.

Nollet recuerda que, en un experimento anterior, cuando intentó hacer descender la presión mientras estaba hirviendo el agua, ésta descendía para volver a recuperarse en seguida. La explicación es que la presión se recupera porque entra algún fluido por los poros del vidrio, que se ve un tanto retardado en su paso, de modo que eso explicaría que al efectuar las emboladas la presión tardase un tanto en recuperarse. Y también este retraso explicaría en parte que pudiese ejercer presión en la campana a

\footnotetext{
48 Del experimento da cuenta MiddLeton, W. E. K. (1964), The History of the Barometer, Baltimore: The Johns Hopkins Press, pp. 78-82. Véase asimismo S. SHAPIN y S.SCHAFER (1985), Leviathan and the Air-Pump, Princeton, Princeton Univ. Press, pp. 241-255.

49 Las ideas de Mairan sobre la aurora boreal se revisan brevemente en J. MORTON BRIGGS,JR. (1967), «Aurora and Enlightenment. Eighteenth-Century Explanations of the Aurora Borealis», Isis, 58, 491-503. En su tratado, Mairan menciona en apoyo de su idea las experiencias antes referidas en la Academia de Ciencias de París, así como el fenómeno de la suspensión anómala. D'ORTOUs DE MAIRAN, J. (1733), Traité physique et historique de l'aurore boréale, París, pp. 44-50.

50 No hay que identificar este «aire sutil» con el éter cartesiano. Cuando Musschenbroek critica su intervención en la explicación del mecanismo del estacionamiento anómalo, cuida de separar la explicación basada en la «materia sutil de Descartes» de la de aquéllos que sostienen que hay varios tipos de aire con diferentes grados de sutileza. VAN MUSSCHENBROEK, P. (1739), pp. 657-660.

51 De hecho, el fenómeno de ósmosis que relata en la última parte de la memoria, en donde constata que una membrana animal permite pasar el agua y no el alcohol (cuando se supone que las partículas de este último, más evaporable, serían más sutiles), viene en apoyo de esta idea.
} 
pesar de permear sus poros. Y quizás, al mezclarse con el vapor contenido en la campana, pierda también algo de su sutileza.

$\mathrm{Y}$, naturalmente, tampoco duda de que sea este aire el responsable del curioso descenso del mercurio por debajo de su nivel en el barómetro de prueba. En realidad, notó tal efecto al enfriar la campana con esponjas empapadas en agua fría, y el fenómeno sólo duró unos minutos, el tiempo necesario, supone, para que el enfriamiento se hiciese sentir en el barómetro, que estaba más o menos en el centro de la campana.

El caso es que éste es uno de los efectos que, al repetir las experiencias, no se produce con regularidad. Lo que, de acuerdo con Nollet, apunta a la existencia de alguna otra causa que todavía no se ha desvelado. Apunta que ésta podría ser la desigual calidad del vidrio.

\section{EL AIRE SUTIL, LA DUREZA Y LA FLUIDEZ}

Como se dijo en la primera parte de este artículo, los físicos experimentales newtonianos explicaban tanto la cohesión de la materia como la elasticidad, siguiendo lo expuesto por Newton en las Cuestiones de la Óptica, mediante una ontología de acciones a distancia. Quienes, como Nollet, no aceptaban este tipo de interacciones, admitiendo sólo causas estrictamente mecánicas, tenían que recurrir al impacto o a la presión. De modo que allí donde unos suponían una fuerza a distancia, los otros presentaban la presión de un fluido:

«tras Descartes, la regla más generalmente observada ha sido la de tratar de explicar por el choque o por el impulso de fluidos invisibles todo lo que no puede serlo por la acción del aire sensible, o de otros cuerpos de los cuales podemos ver las operaciones» ${ }^{52}$.

$\mathrm{Si}$, por otra parte, el fluido sutil puede atravesar el vidrio, cabe presumir que también penetra a los otros cuerpos sólidos. Como en el caso del vidrio arriba comentado, esta penetración se efectúa con más o menos dificultad según el tamaño y la disposición de los poros. Resulta así que la presión de este aire sutil es responsable también de la solidez y de la fluidez. Los cuerpos más porosos le oponen menos resistencia, y son por consiguiente los que presentan entre sus partes menor adhesión. De este modo, las fuerzas de cohesión resultan proporcionales a la cantidad de aire sutil que contienen los cuerpos en su interior, de la que depende la capacidad de «sostener

52 Nollet, J. A. (1754-65), vol. 2, p. 455. Indica que incluso Newton, en las Cuestiones 18 y siguientes de su Óptica, admite la existencia de un fluido sutil (el éter) que permanecería en el interior de una campana neumática cuando en ésta se hubiese hecho el vacío. NEWTON, I. (1977), Óptica, Intr., trad. y notas de C. Solís, Madrid, Alfaguara, p. 18. Pero el éter de Newton no deja de ser newtoniano, pues tiene un carácter elástico debido a interacciones a distancia entre sus partículas. 
la presión» del aire sutil del exterior del cuerpo. Cuando el cuerpo es fluido, está ampliamente penetrado por la materia sutil, por lo que sus partes están más separadas y prestas a moverse entre sí. Y esto, al margen del calor, que por su acción —como se vio - también puede separar las partes y convertir los sólidos en fluidos; $;{ }^{53}$ no se trataba de una hipótesis gratuita:

«hipótesis por hipótesis, creo que es más seguro razonar con principios mecánicos y bien inteligibles, que apoyarse en novedades [se refiere, naturalmente, a las fuerzas a distancia] que no se presentan como ideas familiares al espíritu» ${ }^{54}$.

Según el principio conocido como «la navaja de Occam», no se debían multiplicar los entes sin necesidad. Este principio era fielmente seguido por Nollet, quien, en lugar de multiplicar los entes, como haría la física experimental «exacta» de la última parte del siglo aceptando la existencia de una serie de fluidos sutiles dotados de propiedades específicas, aumentó las funciones ${ }^{55}$.

Sin embargo, aumentar los entes tenía sus ventajas. Para Nollet, los componentes del aire podían tener cualquier grado de sutileza, al igual que los poros de la materia podían tener diversas anchuras y/u orientaciones. En tales circunstancias, cuantificar, por ejemplo, la densidad del fluido sutil encerrado en un cuerpo resultaba imposible. Otra cosa era suponer que cada función era ejercida por un fluido específico y homogéneo que, dentro de un mismo cuerpo, podían superponerse sin interferirse. Un cuerpo, así, puede tener una cantidad de fluido calorífico y a la vez, de manera independiente, una cantidad de fluido eléctrico.

Pero no se trataba sólo, como se dice a veces, de introducir magnitudes cuantificables. La cuantificación, elemento de control o triunfo de la razón more mathemati-

53 NOLLET, J. A. (1754-65), vol. 2, pp. 456 y ss.

54 Ibid., p. 473.

55 Hasta el primer tercio del siglo XVIII, se pensó, en general, que la materia eléctrica que surgía en efluvios de los cuerpos electrizados por frotamiento era característica de la sustancia del cuerpo en cuestión. Pero con el descubrimiento de la conducción eléctrica por Stephen Gray en 1731, que mostraba que los efluvios eléctricos podían transferirse entre distintos cuerpos, surgió la idea de una materia universal de la electricidad. Éste fue en concreto el parecer de Nollet, quien al presentar su teoría en 1745 atribuyó los fenómenos eléctricos a sendas corrientes, efluentes y afluentes, entre los cuerpos electrizados y los de sus alrededores. Sobre la naturaleza de esta sustancia, Nollet insistió en que no se debían multiplicar los entes sin necesidad, y que convenía tratar de buscarla primero entre aquéllas que ya eran conocidas o supuestas. Y la encontró en la materia del fuego que, envuelto en una materia oleosa o sulfurosa, constituiría el material de los efluvios. HOME, R. W. (1979), subraya esta actitud. Por otra parte, hay que observar que Nollet, y con él sus contemporáneos, pensaban que los efluvios eléctricos permeaban al vidrio, lo cual permitía explicar las experiencias de Haubskee. Por su parte, en la explicación de la botella de Leiden, Franklin supuso al vidrio completamente impermeable al fluido eléctrico. Las teorías eléctricas de ambos se contrastan en YAMAZAKI, E. (1976), «L'Abbé Nollet et Benjamin Franklin», Japanese Studies in the History of Science, $\mathrm{n}^{\circ}$ 15, 37-64. 
co en la última parte de la Ilustración, entró en la física experimental de la mano del instrumentalismo y supuso, como se decía en la cita de Feldman recogida al principio de este trabajo, una verdadera revolución. Pero fue una revolución que acabó con un importante elemento de la vieja física aristotélica que, todavía, había recogido en cierta medida la nueva física de la Revolución Científica: la explicación de los fenómenos por sus causas. El nuevo instrumentalismo, más allá del carácter probabilístico que físicos como Musschenbroek o Nollet —y sus predecesores - habían atribuido a las hipótesis, renunciaba a la búsqueda de las causas en favor de un conocimiento algebraico de las relaciones entre magnitudes medibles ${ }^{56}$. De este modo, Joseph Black pudo medir calores específicos y latentes aún sin manifestarse sobre la naturaleza del calor; y, en la misma línea, Lavoisier y Laplace pudieron colaborar en el alba de la revolución química, aun cuando el primero sostuviese una teoría sustancialista del calor y el otro, al parecer, una estrictamente cinética ${ }^{57}$.

Se ha hablado, tradicionalmente, de que la revolución química fue una revolución «retrasada». También, por seguir empleando el término acuñado más recientemente por Kuhn —en realidad el concepto en historia de la ciencia se remonta a esta misma época - pudo ser una revolución dentro de una revolución, y que llegó a su debido tiempo. Los historiadores de la química han subrayado la importancia que el modelo de la física experimental supuso para las primeras investigaciones de Lavoisier. Pero ¿de qué física experimental se trataba? Era, sin duda, la física experimental de un Coulomb, y ciertamente no la de un Nollet ${ }^{58}$.

56 Véase HeILbRon, J. L. (1979), Electricity in the $17^{\text {th }}$ and $18^{\text {th }}$ Centuries. A Study of Early Modern Physics, Berkeley, Los Ángeles y Londres: Univ. of California Press, pp. 71-73. También HeILBRON, J. L. (1982), pp. 63-65, donde se recogen los primeros capítulos de la obra anterior, seguidos de un resumen del desarrollo de la electricidad. Asimismo HEILBRON, J. L. (1993), Weighing Imponderables and Other Quantitative Science Around 1800, Berkeley, Univ. of California Press, Historical Studies in the Physical and Biological Sciences, Supplement to vol. 24, Part 1, pp. 16-23.

57 Las investigaciones de Lavoisier y Laplace se estudian en el ya clásico trabajo de GUERLAC, H. (1976), «Chemistry as a Branch of Physics: Laplace's Collaborqation with Lavoisier», Historical Studies in the Physical Sciences, 7, 193-276. Más recientemente, SARAIVA, L. M. R. (1997), «Laplace, Lavoisier and the quantification of heat», Physis, 34, Fasc 1-2, 99-137.

58 Donovan, A. (1993), Antoine Lavoisier. Science, Administration, Revolution, Oxford, Blackwell, Cap. 3: «Experimental physics». La caracterización de la disciplina por Donovan, sin embargo, se corresponde más con la «física experimental exacta» que con la que aquí he estado estudiando: ««os instrumentos de investigación hicieron posible la cuantificación analítica, y los físicos experimentales creyeron que la precisa cuantificación llevaría finalmente al rigor matemático.» (p. 49). De este modo, la comparación que hace entre Lavoisier y Nollet en el Capítulo 2 no debería llevarse demasiado lejos. 


\section{CONCLUSIÓN}

Cuando la historia tiene un final feliz, suele justificarse por sí misma, y las conclusiones están servidas. Cuando termina mal, la conclusión suele ser una lección, que por lo general adopta la forma del si-entonces: si Nollet hubiese interpretado correctamente sus experimentos, entonces hubiese concluído esto o aquéllo, o si Nollet hubiese medido esto o aquéllo, entonces habría obtenido tales o cuales resultados, dando un paso en la «buena» dirección.

Desde la perspectiva que da el transcurso del tiempo, sabemos que el final no fue feliz. Pero extraer la moraleja no es tan fácil; quizás por esto apenas se encuentren entre la historiografía comentarios sobre la memoria de Nollet, pese a ser tan conspicuos tanto su autor como su lugar de publicación. Tal como he intentado mostrar (que no analizar), la filosofía experimental de la época tenía sus propios métodos y objetivos, tenía sus instrumentos y todo un programa para la investigación de las causas de los fenómenos naturales. Y este programa se perseguía, experimentos espectaculares de salón aparte, con una gran dosis de seriedad. En cuanto a la moraleja, bien pudiera ser ésta: todo lo que se consigue tiene un precio. En este caso, si se quería avanzar en el estudio de los fenómenos, parece que el precio a pagar era la renuncia al viejo proyecto de conocer sus causas. 